\title{
Conjugate Vaccine
}

National Cancer Institute

\section{Source}

National Cancer Institute. Conjugate Vaccine. NCI Thesaurus. Code C1455.

A category of vaccines created by covalently attaching an antigen from an organism to a carrier protein from the same organism to aid in the delivery of the immunogen. 